\title{
Progresses and Challenges of Omics Studies and Their Impacts in Personalized Medicine
}

\section{Sompop Bencharit*}

Department of Pharmacology, School of Medicine, University of North Carolina at Chapel Hill, CB\#7450, Chapel Hill, NC 27599-7450, USA

In the past decade, we have entered the new era of omics studies that may lead to a true clinical application of personalized medicine [1]. The discovery of DNA structure and more recently the completion of the human genome project allow us to define the relationship between one or more particular genes to a disease. Unfortunately, genetic aberrations of multiple genes and their interactions with complex environmental factors are the major causes of several diseases, including diabetes, hypertension and cancer. More importantly, the expression of these disease-related genes, the stability of mRNA and the final protein product can be modified by a wide-range of physiological processes including single-nucleotide polymorphisms (SNPs), alternate splicings, epigenetic modifications, post-translational modifications, etc. While these processes are a pathophysiological result of host-environment interactions, they present a complex challenge in understanding the disease process.

Recent omics studies, for instance whole transcriptome analysis, microbiomics, proteomics and metabolomics, provide a new insight to unlimited possibilities of host-disease interactions. Typically we use omics methods to compare diseased populations to a control by studying multiple gene products, modifications of genes/proteins, as well as metabolites. These types of study together with the progression of bioinformatics allow us to see a global picture of how each disease affects its host. Each omics study provides us separation between disease and non-disease by alterations of genes, proteins or metabolites, in terms of gene modifications and expression, or protein modifications, or differential metabolites, etc. This may potentially and theoretically lead to gene targets or potential biomarkers that can be used in the future for therapeutic applications.

Epidemiologically, disease can affect populations in a certain way, and certain drugs or interventions may work in reducing mortality and morbidity of certain diseases. However, the progression of omics system biology knowledge together with the emerging personalized medicine, suggest that each treatment or intervention can affect individuals differently [2]. Drugs that effectively cure a condition in one person may not work at all in another individual. This challenge can in theory be understood using current omics tools. While omics studies give us hope to understand individual host-disease interaction, they still have several serious limitations. Technical difficulties, expensive instruments, rare expertise, and others make the use of omics limited. Beside these limitations, the massive amount of data generated from each omics study with often small sample size complicates the data interpretation and restricts the clinical value of omics studies [3]. The majority of omics results are therefore often questioned and rejected by traditional epidemiologists, other clinicians and scholars [3].

There are several recommendations to maximize the value of an omics study. I summarized four important ones here. First, I note the minimization of subject variability and the use of a longitudinal study. Unlike traditional epidemiological studies, in most omics studies, we rely on a small sample size because of expensive techniques. Subject variability can become a confounder masking the true biomarkers and leading to false discovery [4]. A strict subject inclusion/exclusion criterion is very important. Unfortunately, it seems to be neglected in most omics studies. Too often we see studies comparing 10 patients to 10 controls without any specific selection criteria. In addition, most omics studies are cross-sectional in nature. We need to do more longitudinal studies and follow multiple targets in the same subject [5]

Second, utilizing more than one omics technique to validate results is needed. While this point is important, cautious and reasonable application is needed [4]. In proteomic studies, we often see the applications of antibody-based assays to validate mass spectrometry proteomic results. This can present a publication bias towards the proteomic results that can be validated by antibodies. There are not sufficient antibodies for the identification of many proteins and most protein modifications. Perhaps validation of mass spectrometry proteomics can be done using other omics methods. For example, a reduction of protein expression may coincide with the same protein metabolites. In this case a simple metabolomics experiment may be sufficient to validate the proteomic results. Similarly, proteomic results can be complemented by transcriptome data. In addition, a set of proteomic data from one mass spectrometry platform can be confirmed by another platform.

Third, analyzing multiple tissues or body fluids of the same subject with other known clinical markers is beneficial. While we widely apply omics technology to virtually all tissues and body fluids, in a typical omics study we usually see only an analysis of one type of tissue or body fluid [6]. It is imperative to examine diseased tissues, blood, urine, or saliva and, at the same time, compare the results with clinical manifestations. This will allow internal confirmation of your biomarkers.

Finally, relating your omics results with the biology or pathology of disease of interest is necessary. Usually in an omics study, most of the results cannot be explained biologically. This limits future studies and the clinical applications. More importantly it can lead investigators to decide not to publish their data when they cannot make sense of the results. It is therefore important to create a general data deposition

*Corresponding author: Sompop Bencharit, Department of Pharmacology, School of Medicine, University of North Carolina at Chapel Hill, CB\#7450, Chape Hill, NC 27599-7450, USA, E-mail: Sompop_Bencharit@dentistry.unc.edu

Received January 10, 2012; Accepted January 12, 2012; Published January 15 2012

Citation: Bencharit S (2012) Progresses and Challenges of Omics Studies and Their Impacts in Personalized Medicine. J Pharmacogenom Pharmacoproteomics 3:e105. doi:10.4172/2153-0645.1000e105

Copyright: (c) 2012 Bencharit S. This is an open-access article distributed under the terms of the Creative Commons Attribution License, which permits unrestricted use, distribution, and reproduction in any medium, provided the original author and source are credited. 
Citation: Bencharit S (2012) Progresses and Challenges of Omics Studies and Their Impacts in Personalized Medicine. J Pharmacogenom Pharmacoproteomics 3:e105. doi:10.4172/2153-0645.1000e105

Page 2 of 2

independent of publications. This will help in future studies when other investigators may find the same unexplained biomarkers and will eventually enhance future studies.

We are in the exciting age of omics and personalized medicine. I believe that with the rapid progression of technology and careful design of omics studies, in the near future we may be able to truly apply personalized medicine. This may bring a great benefit to future healthcare of the general population.

\section{References}

1. Ginsburg GS, Willard HF (2009) Genomic and personalized medicine: foundations and applications. Transl Res 154: 277-287.

2. Clayton TA, Lindon JC, Cloarec O, Antti H, Charuel C, et al. (2006) Pharmaco- metabonomic phenotyping and personalized drug treatment. Nature 440: 10731077.

3. Eriksson L, Antti H, Gottfries J, Holmes E, Johansson E, et al. (2004) Using chemometrics for navigating in the large data sets of genomics, proteomics, and metabonomics (gpm). Anal Bioanal Chem 380: 419-429.

4. Mischak H, Apweiler R, Banks RE, Conaway M, Coon J, et al. (2007) Clinical proteomics: A need to define the field and to begin to set adequate standards. Proteomics Clin Appl 1: 148-156.

5. Al-Tarawneh SK, Border MB, Dibble CF, Bencharit S (2011) Defining salivary biomarkers using mass spectrometry-based proteomics: a systematic review. OMICS 15: 353-361.

6. Nicholson JK, Holmes E, Lindon JC, Wilson ID (2004) The challenges of modeling mammalian biocomplexity. Nat Biotechnol 22: 1268-1274. 